\title{
Extensive Lesions of Cholinergic Basal Forebrain Neurons Do Not Impair Spatial Working Memory
}

\author{
Joseph A. Vuckovich, Mara E. Semel, and Mark G. Baxter ${ }^{1,2}$ \\ Department of Psychology, Harvard University, Cambridge, Massachusetts 02138, USA
}

\begin{abstract}
A recent study suggests that lesions to all major areas of the cholinergic basal forebrain in the rat (medial septum, horizontal limb of the diagonal band of Broca, and nucleus basalis magnocellularis) impair a spatial working memory task. However, this experiment used a surgical technique that may have damaged cerebellar Purkinje cells. The present study tested rats with highly selective lesions of cholinergic neurons in all major areas of the basal forebrain on a spatial working memory task in the radial arm maze. In postoperative testing, there were no significant differences between lesion and control groups in working memory, even with a delay period of $8 \mathrm{~h}$, with the exception of a transient impairment during the first $2 \mathrm{~d}$ of postoperative testing at shorter delays $(0$ or $2 \mathrm{~h})$. This finding corroborates other results that indicate that the cholinergic basal forebrain does not play a significant role in spatial working memory. Furthermore, it underscores the presence of intact memory functions after cholinergic basal forebrain damage, despite attentional impairments that follow these lesions, demonstrated in other task paradigms.
\end{abstract}

The cognitive functions of the basal forebrain cholinergic system (BFCS) are of considerable interest, given the damage to this system associated with neuropathological conditions such as Alzheimer's disease (Perry et al. 1977, 1978; Bartus et al. 1982, 1985a; Baxter and Gallagher 1997; Bartus 2000). Experimental lesion studies have attempted to address the question of which aspects of cognitive impairment in these human disease states, if any, can be attributed to loss of cholinergic neurons. The present study aims to further clarify the role of the BFCS in spatial cognition.

The extent of acetylcholine loss in aged and demented subjects correlates with the extent of their cognitive impairment (Perry et al. 1977, 1978; Bartus et al. 1982). Experiments on animals have therefore examined the effects of BFCS lesions on spatial learning and memory tasks. These tasks are thought to effectively model the cognitive processes that are usually the first to deteriorate in aged and demented humans (Gallagher and Pelleymounter 1988). However, different BFCS lesion experiments have produced seemingly contradictory results. Early studies suggested an important role for this system in learning and memory. Rats with ibotenic acid lesions of the nucleus basalis magnocellularis/substantia innominata (nBM/SI), the component of the basal forebrain that projects predominantly to neocortex, were impaired on a preoperatively learned delayed nonmatching-tosample (DNMS) radial maze task (Bartus et al. 1985b, 1986). Similarly, lesions to the nBM/SI or medial septum/vertical limb of the diagonal band (MS/VDB; the component of the basal forebrain that projects predominantly to the hippocampus) also impaired learning and performance in spatial working memory in the radial arm maze (Hepler et al. 1985; M'Harzi and Jarrard 1992).

However, studies using ibotenic acid can be questioned because these lesions are not selective for basal forebrain cholinergic neurons (Dunnett et al. 1991; Gallagher and Colombo 1995; Baxter and Murg 2002). For example, deficits in memory after basal forebrain lesions with different excitotoxins do not necessarily correlate with the extent of cholinergic damage (see Dun-

\footnotetext{
'Present address: Department of Experimental Psychology, Oxford University, Oxford, England OX1 3UD, UK.

${ }^{2}$ Corresponding author.

E-MAIL: mark.baxter@psy.ox.ac.uk; FAX +44 (0) 1865310447.

Article and publication are at http://www.learnmem.org/cgi/doi/10.1101/ Im.63504.
}

nett et al. 1991). As a result, an important tool in this line of investigation has been 192 IgG-saporin, an immunotoxin that produces complete and highly selective lesions of cholinergic neurons (Wiley et al. 1991; Wrenn et al. 1999). Studies with this toxin have indicated that the BFCS does not play as central or direct a role in spatial learning and memory as was supposed. Selective cholinergic lesions of either MS/VDB or nBM/SI fail to impair spatial learning in the Morris water maze (Torres et al. 1994; Baxter et al. 1995, 1996; Dornan et al. 1996).

Effects of these lesions on spatial working memory, however, have been more variable. Two studies using intraseptal administration of 192 IgG-saporin reported impairments in radial maze performance in a spatial DNMS procedure; in one study these impairments were delay-dependent (Walsh et al. 1996), and in one they were delay-independent (Shen et al. 1996). However, both studies used midline injections of 192 IgG-saporin that could possibly damage noncholinergic neurons (Torres et al. 1994); neither of these studies provided independent corroboration of intact noncholinergic neurons at the lesion site. McMahan et al. (1997) found that bilateral (as opposed to midline) MS/VDB lesions did not impair performance in a water version of the DNMS radial arm maze task. Rats with similar lesions (in which the integrity of noncholinergic MS/VDB neurons was confirmed) failed to impair memory in a dry-land DNMS task, when rats were trained preoperatively (Chappell et al. 1998), in contrast to the report by Walsh et al. (1996). These latter two studies confirmed that the 192 IgG-saporin lesions produced extensive cholinergic denervation of hippocampus but failed to damage noncholinergic neurons in the MS/VDB. However, a recent study in which cholinergic neuron integrity at the lesion site was verified found impairment in spatial working memory after 192 IgGsaporin lesions of the MS/VDB (Lehmann et al. 2003), suggesting that these lesions can be sufficient to impair spatial memory under some circumstances. Studies examining spatial working memory using rewarded T-maze alternation have not found effects of cholinergic MS/VDB lesions (Pang and Nocera 1999; Kirby and Rawlins 2003). These differences across studies also do not appear to be related to whether the lesion is given before training on the maze task, or afterward. When damage is limited to the MS/VDB, loss of both GABAergic and cholinergic neurons may be required to produce deficits in spatial memory (Pang et al. 2001). 
However, other research has complicated this picture. One study by Wrenn et al. (1999) reported deficits in working memory in the radial maze after intraventricular 192 IgG-saporin lesions, which damaged cholinergic neurons throughout the entire BFCS (MS/VDB, nBM/SI, and the horizontal limb of the diagonal band of Broca [HDB]). Only rats with $>75 \%$ depletion of BF cholinergic cells were impaired. These investigators concluded that only severe cholinergic neuronal loss is sufficient to cause learning and memory deficits; earlier studies that failed to find impairment may not have disabled a sufficient number of cholinergic neurons. Furthermore, this study suggested that lesions limited to hippocampal cholinergic projections are insufficient for impairment; cortical projections must also be damaged. Rats with almost total depletion in one of these structures, but without total BFCS neuron loss $>75 \%$, were unimpaired. Because MS/ VDB cholinergic neurons project primarily to the hippocampus, this finding may account for the lack of impairment seen in earlier studies of 192 IgG-saporin lesions of the MS/VDB.

Nonetheless, the intraventricular injection of toxin in this experiment makes its result difficult to interpret. Injections of this type also damage cerebellar Purkinje cells. Wrenn et al. (1999) reported such damage, although a control experiment that confirmed motor function in all lesion groups suggested that damage to cerebellar neurons was minimal. Later work by Wrenn and Wiley (2001) showed that Purkinje cell lesions, by themselves, do not cause decrements in radial maze performance. In this study, as in the previous one, only rats with both extensive cholinergic basal forebrain lesions and Purkinje cell lesions were
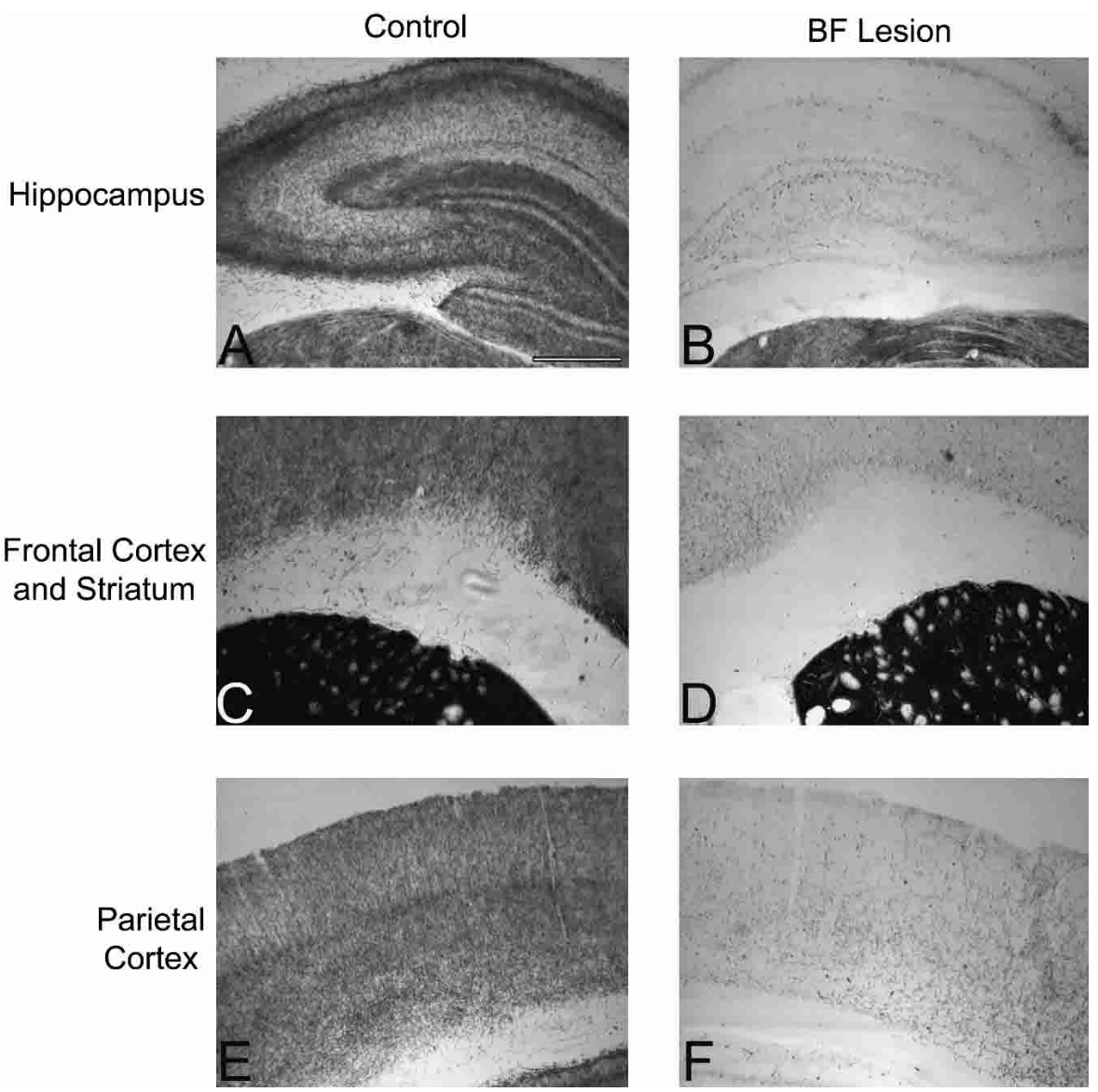

Figure $1 \mathrm{AChE}$ histochemistry. Panels depict $\mathrm{AChE}$ staining in hippocampus $(A, B)$, frontal cortex and striatum $(C, D)$, and parietal cortex $(E, F)$ in a control $(A, C, E)$ and BF-lesioned $(B, D, F)$ rat. Extensive depletion of AChE-positive fibers is apparent in hippocampus and neocortex in the BF-lesioned rat. impaired. Wrenn and Wiley (2001) also showed that spatial memory errors could be predicted based on BFCS cell counts but that adding Purkinje cell counts to the analysis did not increase its predictive power.

Although these results support the view that severe loss of basal forebrain cholinergic neurons impairs spatial working memory, it remains a logical possibility that only the combined loss of cholinergic projections and Purkinje cells will produce impaired performance. The fact that Purkinje cell counts do not make predictions of behavioral performance more accurate does not show that these cells play no role in normal spatial cognition, given that loss of cholinergic neurons and Purkinje cells tended to be highly correlated (Wrenn and Wiley 2001) and other evidence for a role of Purkinje cells in spatial cognition (Goodlett et al. 1992; Gandhi et al. 2000). Only a study of extensive basal forebrain cholinergic lesions without Purkinje cell damage can definitively link such lesions to spatial working memory impairments.

The present study seeks to resolve this issue by examining the effects of extensive and highly selective BFCS lesions (MS/ VDB, HDB, and nBM) on performance in a DNMS task in the radial maze. In contrast to the earlier experiment by Wrenn et al. (1999), in which 192 IgG-saporin was injected into the ventricular system, this experiment uses intraparenchymal injections of neurotoxin. Such injections are not known to damage Purkinje cells (see Heckers et al. 1994). In the DNMS task, rats were allowed to visit four baited arms of a 12-arm radial arm maze prior to a variable delay. At the end of the delay period, free access to all arms of the maze was allowed, but arms visited before the delay period no longer contained food reward. Revisits to arms visited before the delay were scored as retroactive (working memory) errors. Revisits to arms visited after the delay were scored as proactive errors (immediate working memory). Also, four arms of the maze were never baited, and initial entries to these arms (which were only available for entry after the delay) were scored as reference memory errors.

\section{RESULTS}

\section{Subjects}

One rat died of unknown causes before behavioral testing began, and another died after surgery. Six rats did not receive surgeries because they failed to learn the task to criterion. One rat was excluded due to anatomical abnormalities, which appeared to be unrelated to its surgical manipulation, discovered while its brain was being sectioned in preparation for neurobiological analysis. Finally, one rat was excluded on the basis of ChAT immunohistochemistry, which revealed that its MS/VDB and HDB lesions were not complete. Consistent with the immunohistochemical data from this rat, AChE histochemistry revealed preservation of staining in hippocampus and piriform cortex. All sections examined from both lesions and controls exhibited robust parvalbumin staining. Figures 1 and 2 illustrate AChE histochemistry and ChAT and parvalbumin immuno- 

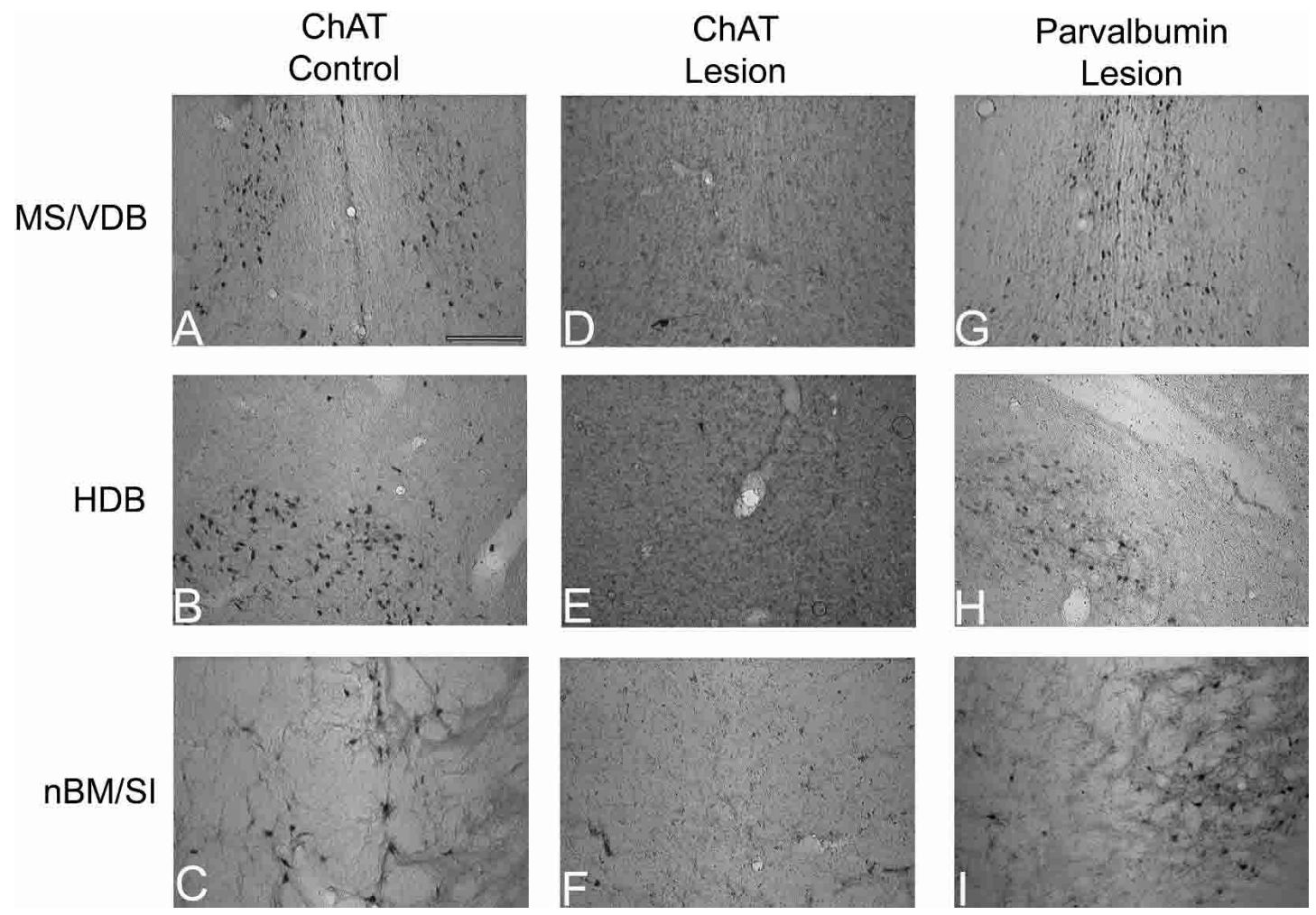

Figure 2 Immunohistochemistry. Panels depict ChAT $(A-F)$ and parvalbumin $(G-l)$ immunohistochemistry in the MS/VDB $(A, D, G), \operatorname{HDB}(B, E, H)$, and $\mathrm{nBM} / \mathrm{SI}(C, F, I)$ of control $(A-C)$ and BF-lesioned $(D-l)$ rats. A loss of cholinergic (ChAT-positive) basal forebrain neurons is apparent in the BF-lesioned rats $(D-F)$, but parvalbumin-immunoreactive neurons are preserved at the lesion site $(G-l)$.

histochemistry in control and BF-lesioned rats. The final number of subjects in each group was eight.

\section{Histological Analysis}

Determinations of mean pixel intensity were made from digital photomicrographs of AChE-stained sections from frontal cortex, parietal cortex, and the CAl, CA3, and DG hippocampal subfields. These values were then normalized to mean densities in striatum. Raw pixel intensity values in striatum (used for normalization) did not differ between the groups (mean \pm SD: control, $215.15 \pm 29.38 ;$ BF lesion, $222.93 \pm 21.43 ; t(14)=-0.61$, $P=0.56)$. Cases that were over-postfixed were equally distributed between the control and lesion groups, so overall values did not differ. The ratio to striatal values, which were not affected by the lesion, allowed comparison of depletion in cases with different amounts of postfixation. Differences in AChE-staining intensity for lesions and controls were determined by comparing the staining in cortical and hippocampal regions (expressed as a ratio to staining in striatum) in a series of unpaired $t$-tests. Lesions had significantly lower ratios than controls for all brain regions examined (Table $1 ; t s(14)>3.66, P s<0.003)$.

This measure of pixel intensity from AChE histochemistry likely underestimates the magnitude of cortical cholinergic denervation; for example, a $32.7 \%$ reduction in staining intensity in frontal cortex does not mean that only $32.7 \%$ of cortical cholinergic input was removed. Because we did not have ChATimmunostained material from all of our rats, and we did not prepare our tissue to permit unbiased stereological counting of cholinergic basal forebrain cells, we were not able to compare our AChE depletions with loss of cholinergic basal forebrain neurons per se. However, we were able to make comparisons with data from another study that used similar methods to measure cortical
AChE densitometry, in rats in which ChAT neuron loss was assessed quantitatively (Wrenn et al. 1999). Their percentage of depletions of AChE densitometry were $28.3 \%$ in dorsolateral frontal cortex, $33.8 \%$ in cingulate cortex, and $38.8 \%$ in hippocampus (all subfields) in their highest-dose 192 IgG-saporin (4 $\mu \mathrm{g})$ group (C.C. Wrenn, pers. comm.). These values compare favorably with those in our study, suggesting any differences in behavioral outcome between the two experiments are probably not due to differences in lesion extent. Furthermore, examination of immunohistochemical material in rats in which it was available confirmed extensive (nearly total) loss of cholinergic (ChAT-immunopositive) neurons in the basal forebrain, with preservation of parvalbumin-immunoreactive neurons, as is typical for these lesions produced in our laboratory (see Cahill and Baxter 2001; De Rosa et al. 2001).

\section{Behavioral Data}

A repeated-measures ANOVA showed that lesions did not alter DNMS performance when all $12 \mathrm{~d}$ of postoperative testing were

Table 1. Densitometry Measurements of AChE Histochemistry (Expressed as Ratio to Striatal Values)

\begin{tabular}{lccccc}
\hline Structure & Control & SD & $\begin{array}{c}\text { BF } \\
\text { lesion }\end{array}$ & SD & $\begin{array}{c}\text { Percentage } \\
\text { decrease }\end{array}$ \\
\hline Frontal cortex & 0.657 & 0.103 & 0.442 & 0.027 & 32.7 \\
Parietal cortex & 0.550 & 0.104 & 0.381 & 0.062 & 30.7 \\
Hippocampal CA1 & 0.589 & 0.091 & 0.403 & 0.037 & 31.6 \\
Hippocampal CA3 & 0.515 & 0.122 & 0.329 & 0.076 & 36.1 \\
Dentate gyrus & 0.603 & 0.127 & 0.324 & 0.111 & 46.2 \\
\hline
\end{tabular}

Mean and SD for each group. 
analyzed together; there was no effect of lesion on retroactive errors (Fig. $3 ; F(1,14)=1.44, P=0.25$ ). As expected, there was a strong main effect of delay; retroactive errors increased with delay for both groups $(F(3,42)=15.07, P<0.0005)$. However, there was no significant delay by lesion interaction $(F(3,42)=0.84$, $P=0.48$ ), indicating that there was no differential effect of lesion at different delays.

Lesion status and delay period did not affect the number of proactive errors committed. There was no main effect of lesion $(F(1,14)=0.03, P=0.86)$ or of delay $(F(3,42)=0.64, P=0.59)$ on this error type. Furthermore, the delay by lesion interaction was not significant for proactive errors $(F(3,42)=1.13, P=0.39)$. BFCS lesions also did not affect the commission of reference memory errors (mean \pm SEM: control, $2.29 \pm 0.22$; lesion, $2.50 \pm 0.24 ; F(1,14)=0.40, P=0.54)$.

In addition to the overall analysis across all $12 \mathrm{~d}$ of postoperative testing, the data were broken into three blocks of four
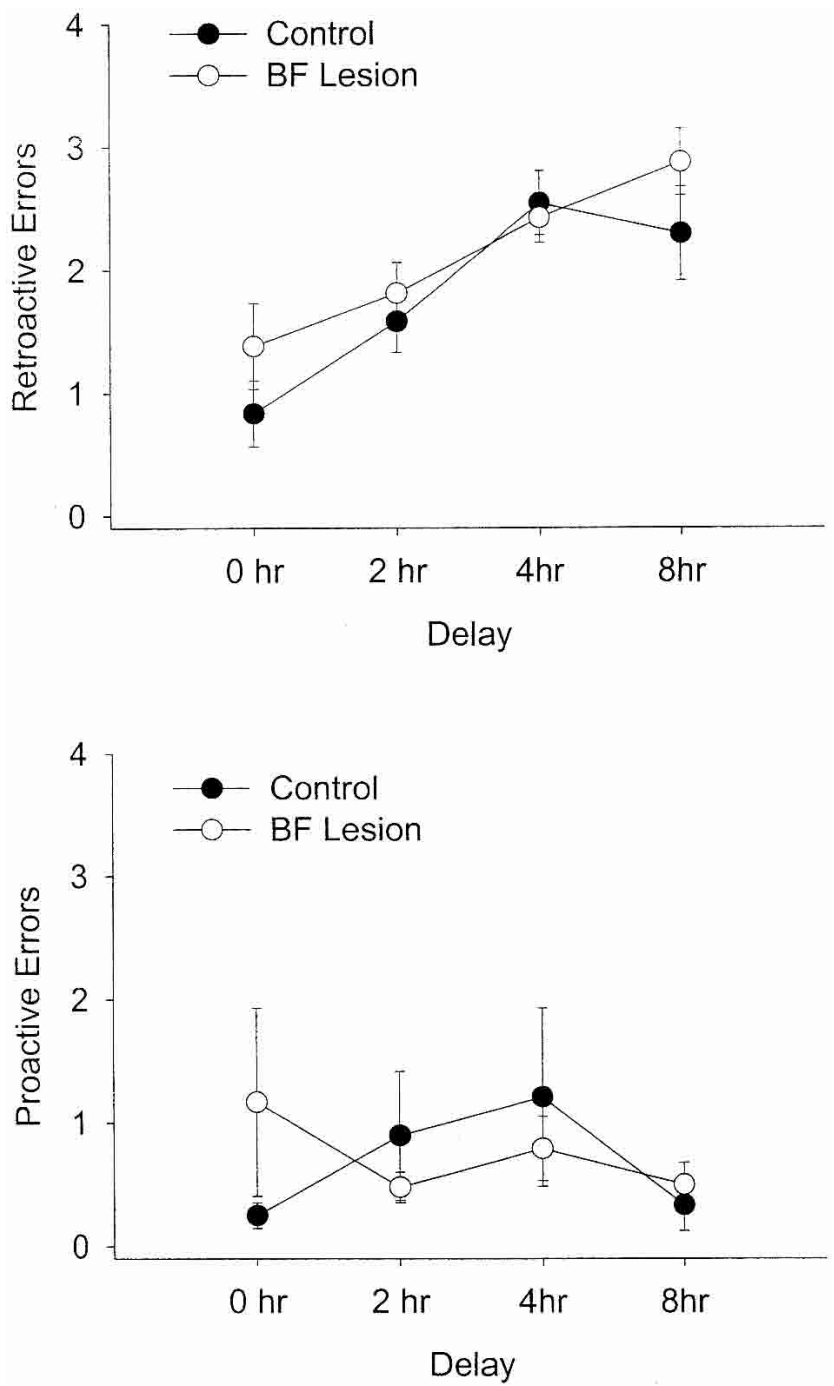

Figure 3 Retroactive and proactive errors at each of the four delays in postoperative testing for control and BF-lesioned rats, across all $12 \mathrm{~d}$ of postoperative testing. Retroactive errors (entries into maze arms visited before the delay) increase as the delay between sample and choice increases, but BF-lesioned rats perform comparably to control rats at each of the four delays. Proactive errors (re-entries into arms visited after the delay) do not vary with delay and also do not differ between control and BF-lesioned rats. testing days each. Each block consisted of $1 \mathrm{~d}$ for each delay period (90 sec, $2 \mathrm{~h}, 4 \mathrm{~h}$, and $8 \mathrm{~h}$ ). Repeated-measures ANOVAs performed on each block showed a main effect of lesion on retroactive errors in block $1(F(1,14)=5.50, P=0.03)$ but not in block $2(F(1,14)=0.09, P=0.77)$ or in block $3(F(1,12)=0.759$, $P=0.40$; Fig. 4). Two rats, one control and one lesion, were excluded from block 3 analysis because they did not complete the DNMS task within the 10-min allotted time on the last day of postoperative testing. The overall analysis of retroactive errors was based on averages obtained from $11 \mathrm{~d}$ of testing for these rats, as opposed to 12 for the other subjects. Analysis by block of proactive errors revealed that there was no effect of lesion on this error type in any block $(F s(1,12$ or 14$)<2.11$, ps $<0.17)$.

Although the analysis by block did reveal a main effect of lesion in block 1 , there was no interaction between DNMS impairment and delay length $(F(3,42)=0.80, P=0.50)$. Thus, the impairment was not delay-dependent. However, unpaired $t$-tests performed for each delay length in block 1 revealed that lesioned rats only made significantly more errors than did controls for the 2-h delay, on the second day of postoperative testing $(P=0.04)$. The difference for the 90-sec delay on the first day of testing approached significance $(P=0.13)$, but lesioned rats and controls performed comparably on the 4 -h $(P=1.00)$ and 8 -h delays $(P=0.32)$. Thus, the apparent memory impairment seen in block 1 resulted almost entirely from poor performance on the first $2 \mathrm{~d}$ of postoperative testing, and disappeared entirely when the delay period became longer.

\section{DISCUSSION}

In the present experiment, rats with extensive BFCS lesions were completely unimpaired in immediate working memory (measured by proactive errors), and relatively unimpaired in working memory for spatial information held across delays. There was no overall differential impairment of working memory in lesioned rats at long delays, but rather only a transient effect of lesion during the first $2 \mathrm{~d}$ of postoperative testing.

These results indicate that extensive basal forebrain cholinergic lesions, by themselves, do not necessarily induce severe memory disturbances of the kind reported in other recent studies (Wrenn et al. 1999; Wrenn and Wiley 2001). Several differences between the current experiment and these other studies of extensive lesions should be noted. First, a six-arm radial maze was used in both previous studies, whereas a 12 -arm maze was used in the experiment reported here. Given that our DNMS task required rats to keep track of more spatial locations, one might expect it to be more sensitive to a lesion-induced memory impairment, but no impairment was found. Furthermore, the present study tested rats at much longer delays, ranging from $90 \mathrm{sec}$ to $8 \mathrm{~h}$; studies showing impairment used very short delays $(\leq 30$ sec). Again, the longer delays in the task reported here would presumably make the DNMS procedure more demanding of spatial memory and thus more sensitive to lesion-induced impairments. However, we presented delays to our rats in a different manner from that of Wrenn et al. (1999): Our rats were given four locations to be remembered before the delay, whereas Wrenn et al. (1999) imposed a delay (from 5 to $30 \mathrm{sec}$ ) after each choice, before allowing the next choice to be made. Hence, the tasks may also differ in the way in which working memory is taxed, and the time course across which memories must be retained. It is possible that allowing rats to acquire a list of spatial locations and then retain it over a long time interval exerts less demand on the BFCS (and its cortical targets) than does continuously updating a list of locations over seconds or minutes (see Turchi and Sarter 2000). Parametric manipulation of this variable within a single study may shed greater light on this issue. 

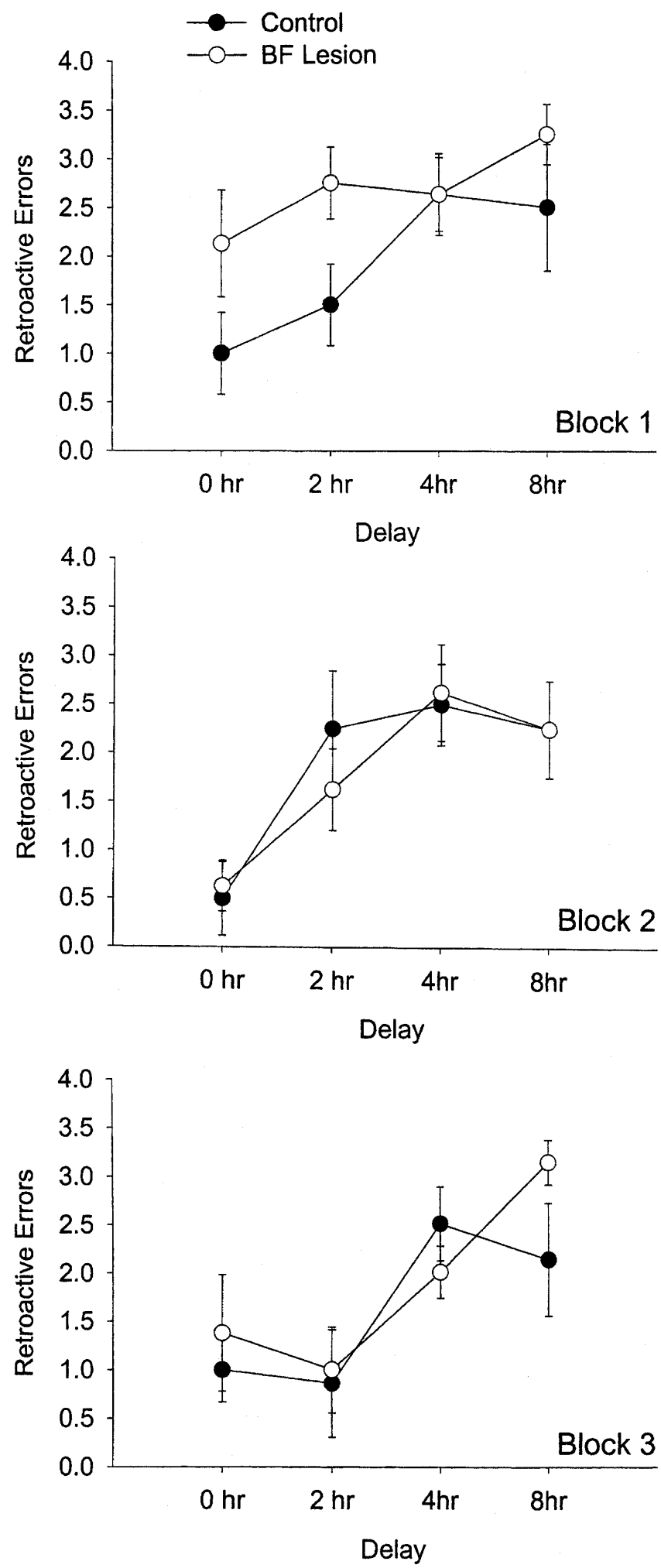

Figure 4 Retroactive errors at each of the four delays, broken down for the three blocks of postoperative testing. An effect on the first $2 \mathrm{~d}$ of postoperative testing (the first two delays of the first block) is apparent, whereas performance of control and BF-lesioned rats does not differ appreciably on any of the other days of testing.
Another possibility is that there are differences in the information available to the rats in solving the radial maze tasks in different studies. Specifically, the radial maze used by Wrenn et al. (1999) had clear arms but opaque covers on each arm, so the rats may have had limited views of extramaze cues depending on where they were in the maze (C.C. Wrenn, pers. comm.). In contrast, our maze was located inside a square test area surrounded by curtains, with a few discrete and salient visual cues. These considerations may affect whether rats use a "cognitive map," path integration information, or memory for egocentric body movement (or some combination thereof) to remember locations they have visited in the radial maze. Differences in information available to use for navigation may also affect the attentional requirements of spatial tasks (a point returned to in a later paragraph). These factors also reflect a potential source of differences between experiments that may need to be taken into account in making cross-study comparisons. A similar explanation might apply to situations in which lesions limited to MS/ VDB cholinergic neurons do or do not impair spatial memory (Chappell et al. 1998; Lehmann et al. 2003).

We verified the completeness of our cholinergic lesions by using AChE densitometry methods similar to those of Wrenn et al. (1999). Our lesions were similar in extent to those reported by Wrenn et al. (1999), which were produced by the highest $(4 \mu \mathrm{g})$ dose of intraventricular 192 IgG-saporin. Immunohistochemical observations indicated near-total depletion of cholinergic neurons in the brains of lesioned animals; furthermore, our depletions of cortical AChE are similar in magnitude to those reported by Wrenn et al. (1999) for their rats with working memory impairment. Thus, although the lesions were produced by different methods (intraventricular versus intraparenchymal injection of 192 IgG-saporin), it seems unlikely that differences in cholinergic lesion extent can account for the different outcomes of our two studies. Thus, it is possible that conjoint damage to cerebellar neurons and basal forebrain cholinergic neurons impairs spatial memory more severely than lesions of either population alone (Wrenn et al. 1999; Wrenn and Wiley 2001) although studies aimed at testing this hypothesis explicitly are needed.

Notably, studies using nonselective lesions of basal forebrain neurons (e.g., with ibotenic acid) in both the MS/VDB and NBM have demonstrated deficits in spatial memory in the radial maze with much smaller reductions in cortical cholinergic innervation ( $\sim 33 \%$, measured by ChAT radioenzymatic assay) than those produced by the 192 IgG-saporin lesions, demonstrating the sensitivity of the radial maze task to basal forebrain damage (see Knowlton et al. 1985). Thus, as noted for other spatial memory tasks, deficits in performance caused by neurotoxic basal forebrain lesions cannot be explained entirely by loss of cholinergic neurons.

We did note a temporary lesion-related deficit in the first block of postoperative testing. BF-lesioned rats were impaired in working memory during the first $2 \mathrm{~d}$ of postoperative testing, on 90-sec and 2-h delays. It is possible that this impairment reflects an initial impairment in working memory. Because performance on retroactive errors did not differ by the third day of testing (on which an even longer delay was presented), or on subsequent days of testing, the deficit may have recovered very rapidly or been attenuated by practice at short delays. This contrasts with enduring deficits reported in other studies (Walsh et al. 1996; Wrenn et al. 1999). It is also possible that the lesioned rats simply did not remember the requirements of the DNMS task as well as did the controls. They might have forgotten the win-shift principle, for example. Comparable analyses for reference memory errors and proactive errors revealed no main effect of lesion on the first $2 \mathrm{~d}$ of postoperative testing, although lesioned rats scored numerically higher than did controls on both measures on both of these days (e.g., proactive errors on day 1 of 
postoperative testing, mean \pm SEM: control, $0.25 \pm 0.16$; BFlesion, $1.38 \pm 0.73 ; t(14)=1.5, P=0.16)$. This would reflect some kind of general retrograde amnesia or disruption of motivation rather than a deficit of spatial working memory per se. Our data do not allow us to distinguish between these possibilities, but at the very least, spatial working memory capacity across long delays appears to be intact overall and not subject to enduring impairments after extensive basal forebrain cholinergic lesions.

Attentional defects are reliably observed after selective loss of basal forebrain cholinergic neurons (McGaughy et al. 1996; Sarter et al. 1997; McGaughy and Sarter 1998; Sarter and Bruno 1999). It is interesting to note that despite these attentional impairments (which were not measured directly in the present study, but for impairments in attention after similar basal forebrain lesions, see Baxter et al. 1999), our rats demonstrated intact spatial working memory, even at long $(8 \mathrm{~h})$ delays. It is not obvious how, in the presence of disrupted attentional function, rats could learn which four of the 12 arms were visited before the delay period and retain this information across long delay periods. This finding, as well as other reports of intact learning and memory functions after damage to basal forebrain cholinergic neurons, suggests that impairments in attention after basal forebrain cholinergic lesions must exert relatively circumscribed effects on the overall efficiency of cognitive processing. That is, these lesions might be better characterized as disrupting the capacity to regulate attentional resources rather than the capacity to attend to stimuli per se (Baxter and Chiba 1999). If spatial memory tasks were designed to tax attentional resources more severely (e.g., by limiting the amount of spatial information available to navigate or by requiring the rat to attend to a subset of cues for navigation), it seems likely that deficits would emerge after basal forebrain lesions. However, the presence of dissociations between mnemonic and attentional performance after basal forebrain cholinergic damage suggests that many memory processes, including consolidation, storage, and retrieval, as well as encoding, can operate perfectly normally in the absence of cholinergic input.

\section{MATERIALS AND METHODS}

\section{Subjects}

Twenty-six male hooded Long-Evans rats (Taconic, Germantown, NY) were housed separately in cages on a 12-h light/12-h dark cycle beginning at 8:00 a.m. Throughout behavioral testing, animals were kept on food restriction and maintained at $85 \%$ of their free-feeding weight. They were placed back on ad libitum food prior to surgery and during the recovery period after surgery.

\section{Surgery}

Following pretraining to criterion, rats were assigned to lesion (final $n=8$ ) or control (final $n=8$ ) groups based on the number of trials they required to reach criterion in pretraining on the DNMS task. The mean number of trials required to reach criterion did not differ significantly between the two matched groups. All surgery conditions were identical except that control rats received microinjections of $0.1 \mathrm{M}$ phosphate-buffered saline, whereas lesioned rats received injections of 192 IgG-saporin $(0.12$ or $0.15 \mu \mathrm{g} / \mu \mathrm{L}$ in $0.1 \mathrm{M}$ phosphate-buffered saline; Chemicon). The immunotoxin used in this study was prepared from two different batches, which did not differ in potency at their final concentration $(0.12$ or $0.15 \mu \mathrm{g} / \mu \mathrm{L})$ based on pilot experiments in our laboratory.

Surgical procedures were similar to those outlined in Baxter et al. (1995) and De Rosa et al. (2001). Each rat was anaesthetized with ketamine $(80 \mathrm{mg} / \mathrm{kg}$, intramuscularly) and xylazine $(5 \mathrm{mg} /$ $\mathrm{kg}$, intramuscularly). Anesthesia was supplemented as necessary throughout surgery, and surgery was conducted under aseptic conditions.
The coordinates for all injections were measured from bregma and determined by using the Paxinos and Watson (1986) rat brain atlas. Four injections were placed in the MS/VDB at $\mathrm{AP}=+0.45 \mathrm{~mm}$ and $\mathrm{ML}= \pm 0.6 \mathrm{~mm}$, at two depths on each side: $\mathrm{DV}=-7.8 \mathrm{~mm}$ and $-6.2 \mathrm{~mm}$. The $\mathrm{DV}-7.8 \mathrm{~mm}$ injection volume was $0.3 \mu \mathrm{L}$, and the DV $-6.2 \mathrm{~mm}$ injection volume was 0.2 $\mu \mathrm{L}$. All injections into the MS/VDB were delivered at a rate of 0.05 $\mu \mathrm{L} / \mathrm{min}$. Each rat also received two injections into the HDB at $\mathrm{AP}=-0.15 \mathrm{~mm}, \mathrm{ML}= \pm 1.8 \mathrm{~mm}$, and $\mathrm{DV}=-8.7 \mathrm{~mm} ; 0.3 \mu \mathrm{L}$ was delivered at $0.1 \mu \mathrm{L} / \mathrm{min}$. Finally, there were four injections to $\mathrm{nBM} / \mathrm{SI}$, at $\mathrm{AP}=-0.75 \mathrm{~mm}, \mathrm{DV}=-8.1 \mathrm{~mm}($ at $\mathrm{ML}= \pm 3.3 \mathrm{~mm})$, and $\mathrm{DV}=-7.8 \mathrm{~mm}($ at $\mathrm{ML}= \pm 2.3 \mathrm{~mm})$. These consisted of 0.2 $\mu \mathrm{L}$ infused at $0.1 \mu \mathrm{L} / \mathrm{min}$.

\section{Behavioral Training and Testing}

Rats were trained to perform a spatial working memory task on a 12 -arm radial maze. The maze was surrounded by black curtains forming a square $\left(7^{\prime} \times 7^{\prime}\right)$ enclosure. Each curtain contained a different large $\left(2^{\prime} \times 3^{\prime}\right)$ black-and-white poster as a visual cue. No other objects in the testing room were visible. The rats were observed through a camera suspended above the center of the maze and connected to a television monitor.

Rats were habituated to the radial maze for $10 \mathrm{~d}$ and then began pretraining on the DNMS task. Each trial of pretraining proceeded as follows. Four arms of the maze were randomly selected to serve as reference memory arms and were not baited on any trial; these were the same for all rats. Hence, across sessions, the rats were to learn that entries into these arms were never reinforced. The doors leading to these arms were closed during the predelay period. Of the remaining eight arms, four were baited at the beginning of the trial and had their doors open; four others had their doors closed. The four baited arms open during the predelay period were chosen randomly on each day of testing. After the rat had gone to the ends of each of the four open baited arms, it was removed for a delay of $90 \mathrm{sec}$. When the rat re-entered the maze after the 90-sec delay, all doors were open and only the four previously unbaited arms contained food rewards. The trial finished when the rat had gone to the ends of all four newly baited arms or $10 \mathrm{~min}$ had elapsed. Each rat had one trial per day with $\sim 2 \mathrm{~d}$ off per week.

During the pretraining DNMS trials, each rat's performance was scored for working memory errors and reference memory errors. Working memory errors consisted of entries into an arm baited before the delay (retroactive errors) and entries into an arm previously visited during the postdelay phase (proactive errors). First entries into any of the four never-baited arms were scored separately as reference memory errors. Subsequent entries into any of these arms were scored as additional proactive errors. Rats continued with pretraining until they reached criterion levels of performance, defined as three consecutive days with mean less than one working memory errors.

Before postoperative testing, rats were allowed 2 weeks to recover from surgery. Postoperative testing involved a procedure identical to that of pretraining, except that the length of the delay was varied. Rats were tested on 90-sec, 2-h, 4-h, and 8-h delays. Again, they had one trial per day. The delay periods were initially presented in ascending order for the first $4 \mathrm{~d}$ of postoperative testing (i.e., block 1 of postoperative testing) and then randomly varied for subsequent blocks of postoperative testing. There were 12 total days of postoperative testing, so that each rat was tested three times on each delay. Scoring was the same as in pretraining.

\section{Histological Analysis}

After completion of radial maze testing, all rats were killed with an overdose of Nembutal $(0.8 \mathrm{~mL})$ and were transcardially perfused with ice-cold $0.9 \%$ normal saline $(5 \mathrm{~min}$ ) followed by icecold $4 \%$ paraformaldehyde $(20 \mathrm{~min})$ at a flow rate of $\sim 18 \mathrm{~mL} /$ min. All brains were postfixed in $4 \%$ paraformaldehyde and then transferred to $20 \%$ sucrose in phosphate-buffered saline. The brains remained in this solution for 4 to $6 \mathrm{~d}$, after which they were cut into sets of six $60-\mu \mathrm{m}$ coronal sections on a freezing 
sliding microtome. As a result of experimenter error, half of the brains in each group were left in the postfix for 1 week rather than $2 \mathrm{~h}$. This resulted in abnormally light AChE staining in these brains-a result that was taken into account in our AChE fiber density counts (see Results).

Several different neurobiological measures were used to assess the completeness and selectivity of the lesions. AChE histochemistry was performed on sections taken from all areas between and including the frontal cortex and the caudal hippocampus in every rat. The procedure for AChE staining was essentially that outlined in Paxinos and Watson (1986). Photomicrographs of AChE-stained sections were analyzed by using the software package Image (National Institutes of Health). Estimates of mean pixel intensity were obtained for frontal cortex, parietal cortex, and the CA1, CA3, and dentate gyrus (DG) hippocampal subfields. These values were then normalized to pixel densities in striatum to eliminate confounds that could arise from different intensities of AChE stain in different brains-in particular, from the notably lighter stains obtained from all brains left in postfix for a week. This procedure for the quantitative evaluation of AChE-staining fiber density is similar to methods used in other BFCS lesion studies (see Dougherty et al. 1998; Wrenn et al. 1999).

To obtain a qualitative assessment of the completeness and selectivity of the immunolesions, ChAT and parvalbumin immunocytochemistry were performed on sections through the basal forebrain taken from a subset of the rats (those that were postfixed for $2 \mathrm{~h}$; the extended postfixation of the remainder of the brains precluded their immunohistochemical analysis). Immunostaining was done by using avidin-biotin complex methods discussed elsewhere (Cahill and Baxter 2001). ChAT immunohistochemistry was carried out with monoclonal anti-ChAT antibodies (goat monoclonal anti-ChAT, Vector Labs). Parvalbumin immunostaining was completed with monoclonal anti-parvalbumin antibodies (mouse monoclonal anti-parvalbumin, Sigma).

\section{ACKNOWLEDGMENTS}

We thank Morgan Barense, Agnieszka Janisiewicz, Steve Kim, and Emily Murphy for technical assistance. We are also grateful to Craige Wrenn for providing raw data on AChE values from his experiment (Wrenn et al. 1999) and for his very helpful comments on an earlier version of this manuscript. This study was supported in part by the Harvard College Research Program. M.G.B. is an Alfred P. Sloan Research Fellow.

The publication costs of this article were defrayed in part by payment of page charges. This article must therefore be hereby marked "advertisement" in accordance with 18 USC section 1734 solely to indicate this fact.

\section{REFERENCES}

Bartus, R.T. 2000. On neurodegenerative diseases, models, and treatment strategies: Lessons learned and lessons forgotten a generation following the cholinergic hypothesis. Exp. Neurol. 163: $495-529$.

Bartus, R.T., Dean III, R.L., Beer, B., and Lippa, A.S. 1982. The cholinergic hypothesis of geriatric memory dysfunction. Science 217: 408-417.

Bartus, R.T., Dean III, R.L., Pontecorvo, M.J., and Flicker, C. 1985a. The cholinergic hypothesis: A historical overview, current perspective, and future directions. Ann. N.Y. Acad. Sci. 444: 332-358.

Bartus, R.T., Flicker, C., Dean III, R.L., Pontecorvo, M., Figueiredo, J.C., and Fisher, S.K. 1985b. Selective memory loss following nucleus basalis lesions: Long term behavioral recovery despite persistent cholinergic deficiencies. Pharmacol. Biochem. Behav. 23: 125-135.

Bartus, R.T., Pontecorvo, M.J., Flicker, C., Dean III, R.L., and Figueiredo, J.C. 1986. Behavioral recovery following bilateral lesions of the nucleus basalis does not occur spontaneously. Pharmacol. Biochem. Behav. 24: 1287-1292.

Baxter, M.G. and Chiba, A.A. 1999. Cognitive functions of the basal forebrain. Curr. Opin. Neurobiol. 9: 178-183.

Baxter, M.G. and Gallagher, M. 1997. Cognitive effects of selective loss of basal forebrain cholinergic neurons: Implications for cholinergic therapies of Alzheimer's disease. In Pharmacological treatment of Alzheimer's disease: Molecular and neurobiological foundations (eds. J.D.
Brioni and M.W. Decker), pp. 87-103. Wiley, New York.

Baxter, M.G. and Murg, S.L. 2002. The basal forebrain cholinergic system and memory: Beware of dogma. In Neuropsychology of memory, 3rd ed. (eds. L.R. Squire and D.L. Schacter), pp. 425-436. Guilford Press, New York.

Baxter, M.G., Bucci, D.J., Gorman, L.K., Wiley, R.G., and Gallagher, M. 1995. Selective immunotoxic lesions of basal forebrain cholinergic cells: Effects on learning and memory in rats. Behav. Neurosci. 109: 714-722.

Baxter, M.G., Bucci, D.J., Sobel, T.J., Williams, M.J., Gorman, L.K., and Gallagher, M. 1996. Intact spatial learning following lesions of basal forebrain cholinergic neurons. NeuroReport 7: 1417-1420.

Baxter, M.G., Bucci, D.J., Holland, P.C., and Gallagher, M. 1999. Impairments in conditioned stimulus processing and conditioned responding after combined selective removal of hippocampal and cortical cholinergic input. Behav. Neurosci. 113: 486-495.

Cahill, J.F.X. and Baxter, M.G. 2001. Cholinergic and noncholinergic septal neurons modulate strategy selection in spatial learning. Eur. J. Neurosci. 14: 1856-1864.

Chappell, J., McMahan, R., Chiba, A., and Gallagher, M. 1998. A re-examination of the role of basal forebrain cholinergic neurons in spatial working memory. Neuropharmacology 37: 481-487.

De Rosa, E., Hasselmo, M.E., and Baxter, M.G. 2001. Contribution of the cholinergic basal forebrain to proactive interference from stored odor memories during associative learning in rats. Behav. Neurosci. 115: $314-327$

Dornan, W.A., McCampbell, A.R., Tinkler, G.P., Hickman, L.J., Bannon, A.W., Decker, M.W., and Gunther, K.L. 1996. Comparison of site-specific injections into the basal forebrain on water maze and radial arm maze performance in the male rat after immunolesioning with 192 IgG saporin. Behav. Brain Res. 82: 93-101.

Dougherty, K.D., Turchin, P.I., and Walsh, T.J. 1998. Septocingulate and septohippocampal cholinergic pathways: Involvement in working/episodic memory. Brain Res. 810: 59-71.

Dunnett, S.B., Everitt, B.J., and Robbins, T.W. 1991. The basal forebrain-cortical cholinergic system: Interpreting the functional consequences of excitotoxic lesions. Trends Neurosci. 14: 494-501.

Gallagher, M. and Colombo, P.J. 1995. Ageing: The cholinergic hypothesis of cognitive decline. Curr. Opin. Neurobiol. 5: 161-168.

Gallagher, M. and Pelleymounter, M.A. 1988. Spatial learning deficits in old rats: A model for memory decline in the aged. Neurobiol. Aging 9: $549-556$.

Gandhi, C.C., Kelly, R.M., Wiley, R.G., and Walsh, T.J. 2000. Impaired acquisition of a Morris water maze task following selective destruction of cerebellar Purkinje cells with OX7-saporin. Behav. Brain Res. 109: 37-47.

Goodlett, C.R., Hamre, K.M., and West, J.R. 1992. Dissociation of spatial navigation and visual guidance performance in Purkinje cell degeneration (pcd) mutant mice. Behav. Brain Res. 47: 129-141.

Heckers, S., Ohtake, T., Wiley, R.G., Lappi, D.A., Geula, C., and Mesulam, M.-M. 1994. Complete and selective cholinergic denervation of rat neocortex and hippocampus but not amygdala by an immunotoxin against the p75 NGF receptor. J. Neurosci. 14: $1271-1289$

Hepler, D.J., Olton, D.S., Wenk, G.L., and Coyle, J.T. 1985. Lesions in nucleus basalis magnocellularis and medial septal area of rats produce qualitatively similar memory impairments. J. Neurosci. 5: $866-873$.

Kirby, B.P. and Rawlins, J.N.P. 2003. The role of the septo-hippocampal cholinergic projection in T-maze rewarded alternation. Behav. Brain Res. 143: $41-48$.

Knowlton, B.J., Wenk, G.L., Olton, D.S., and Coyle, J.T. 1985. Basal forebrain lesions produce a dissociation of trial-dependent and trial-independent memory performance. Brain Res. 345: 315-321.

Lehmann, O., Grottick, A.J., Cassel, J.-C., and Higgins, G.A. 2003. A double dissociation between serial reaction time and radial maze performance in rats subjected to 192 IgG-saporin lesions of the nucleus basalis and/or the septal region. Eur. J. Neurosci. 18: 651-666.

McGaughy, J. and Sarter, M. 1998. Sustained attention performance in rats with intracortical infusions of 192 IgG-saporin-induced cortica cholinergic deafferentation: Effects of physostigmine and FG 7142. Behav. Neurosci. 112: 1519-1525.

McGaughy, J., Kaiser, T., and Sarter, M. 1996. Behavioral vigilance following infusions of $192 \mathrm{IgG}$-saporin into the basal forebrain: Selectivity of the behavioral impairment and relation to cortical AChE-positive fiber density. Behav. Neurosci. 110: 247-265.

McMahan, R.W., Sobel, T.J., and Baxter, M.G. 1997. Selective immunolesions of hippocampal cholinergic input fail to impair spatial working memory. Hippocampus 7: 130-136.

M'Harzi, M. and Jarrard, L.E. 1992. Effects of medial and lateral septal lesions on acquisition of a place and cue radial maze task. Behav. 
Vuckovich et al.

Brain Res. 49: 159-165.

Pang, K.C.H. and Nocera, R. 1999. Interactions between 192-IgG saporin and intraseptal cholinergic and GABAergic drugs: Role of cholinergic medial septal neurons in spatial working memory. Behav. Neurosci. 113: $265-275$.

Pang, K.C.H., Nocera, R., Secor, A.J., and Yoder, R.M. 2001. GABAergic septohippocampal neurons are not necessary for spatial memory. Hippocampus 11: 814-827.

Paxinos, G. and Watson, C. 1986. The rat brain in stereotaxic coordinates, 2nd ed. Academic Press, San Diego, CA.

Perry, E.K., Perry, R.H., Blessed, G., and Tomlinson, B.E. 1977. Necropsy evidence of central cholinergic deficits in senile dementia. Lancet i: 189 .

Perry, E.K., Tomlinson, B.E., Blessed, G., Bergmann, K., Gibson, P.H., and Perry, R.H. 1978. Correlation of cholinergic abnormalities with senile plaques and mental test scores in senile dementia. Brit. Med. J. 2: $1457-1459$.

Sarter, M. and Bruno, J.P. 1999. Abnormal regulation of corticopetal cholinergic neurons and impaired information processing in neuropsychiatric disorders. Trends Neurosci. 22: 67-74.

Sarter, M., Bruno, J.P., and Himmelheber, A.M. 1997. Cortical acetylcholine and attention: Neuropharmacological and cognitive principles directing treatment strategies for cognitive disorders. In Pharmacological treatment of Alzheimer's disease: Molecular and neurobiological foundations (eds. J.D. Brioni and M.W. Decker), pp. 105-128. Wiley, New York.

Shen, J., Barnes, C.A., Wenk, G.L., and McNaughton, B.L. 1996. Differential effects of selective immunotoxic lesions of medial septal cholinergic cells on spatial working and reference memory. Behav. Neurosci. 110: 1181-1186.

Torres, E.M., Perry, T.A., Blokland, A., Wilkinson, L.S., Wiley, R.G. Lappi, D.A., and Dunnett, S.B. 1994. Behavioural, histochemical and biochemical consequences of selective immunolesions in discrete regions of the basal forebrain cholinergic system. Neuroscience 63: $95-122$.

Turchi, J. and Sarter, M. 2000. Cortical cholinergic inputs mediate processing capacity: Effects of 192 IgG-saporin-induced lesions on olfactory span performance. Eur. J. Neurosci. 12: 4505-4514.

Walsh, T.J., Herzog, C.D., Gandhi, C., Stackman, R.W., and Wiley, R.G. 1996. Injection of 192 IgG-saporin into the medial septum produces cholinergic hypofunction and dose-dependent working memory deficits. Brain Res. 726: 69-79.

Wiley, R.G., Oeltmann, T.N., and Lappi, D.A. 1991. Immunolesioning: Selective destruction of neurons using immunotoxin to rat NGF receptor. Brain Res. 562: 149-153.

Wrenn, C.C. and Wiley, R.G. 2001. Lack of effect of moderate Purkinje cell loss on working memory. Neuroscience 107: 433-445.

Wrenn, C.C., Lappi, D.A., and Wiley, R.G. 1999. Threshold relationship between lesion extent of the cholinergic basal forebrain in the rat and working memory impairment in the radial maze. Brain Res. 847: 284-298.

Received May 30, 2003; accepted in revised form November 17, 2003.

\section{Learning \& Memory}




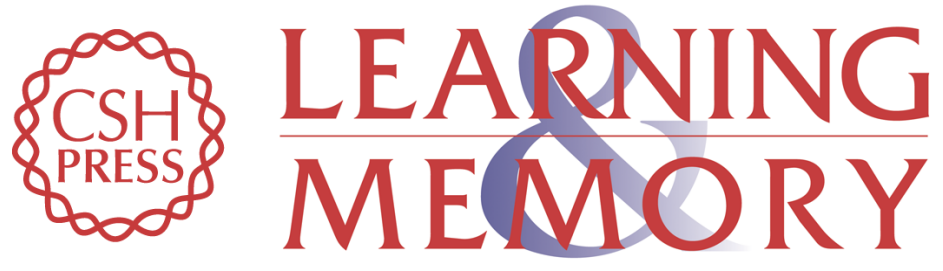

\section{Extensive Lesions of Cholinergic Basal Forebrain Neurons Do Not Impair Spatial Working Memory}

Joseph A. Vuckovich, Mara E. Semel and Mark G. Baxter

Learn. Mem. 2004, 11:

Access the most recent version at doi:10.1101/lm.63504

References This article cites 39 articles, 3 of which can be accessed free at:

http://learnmem.cshlp.org/content/11/1/87.full.html\#ref-list-1

License

Email Alerting Receive free email alerts when new articles cite this article - sign up in the box at the Service top right corner of the article or click here. 